alternative machine a few shillings. Personally I use an exercising machine which is on the market and is of American manufacture. ${ }^{1}$ It is very perfectly constructed and answers my purpose admirably. It consists of a long rubber cord terminating at each extremity in handles. This cord passes round three pulleys which can be variously grouped and attached by hooks to the wall or to the rails of the bed. We can thus by a proper arrangement of the apparatus oppose resistance to almost any movement of the patient either lying or standing or sitting.

As mechanically resisted movements produce a more marked effect upon the patient than the Nauheim ones, even greater precautions must be taken in administering them. It must be made an absolute rule that the exercises are never to be given except in the presence of a medical man until the treatment has so far advanced that it has been ascertained not only that it is doing good, but also exactly how much mechanical work the patient can stand.

The first exercises must be tentative, using the mildest movements and the lightest resistance. In order that the physician should obtain an intelligent idea of the effect produced the following points should be observed: (1) the pulse-rate must be taken before, during, and after the exercises; (2) from time to time during the course of the treatment a sphygmographic tracing of the pulse must be made before and after the exercises; (3) the rate of respiration must be observed and the effect of the movements upon it; (4) the position of the apex beat before and after the treatment; and (5) the area of cardiac dulness must be noted before and after the exercises. To estimate variations in the size of the heart I am accustomed to depart somewhat from traditional methods and attempt to measure the heart in its longitudinal and transverse diameters. The former may be roughly taken to correspond to the distance of the apex beat from the right limit of cardiac dulness taken at the level of the fourth costal cartilage, and the latter to the extent of the dulness along a line drawn from the xiphoid to the middle point of the left clavicle. In order to secure accuracy we must reduce as much as we can the number of occasions on which error is possible. We have here the advantage of two easily estimated points to measure from, leaving only two lines to be made out by percussion. As an additional precaution it will be as well to note the position of the apex beat with respect to the left midclavicular line and also the distance of the left limit of cardiac dulness from the mid-sternal line.

Having thus observed the effect produced by the exercises upon the patient the physician will be in a position to prescribe those suitable for the particular case he is about to treat, the rapidity with which they are to be executed, and the amount of resistance to be used. During the exercises he will be on the watch for and note any symptom, such as dyspnca, lividity, pallor, faintness, or giddiness, which would show that the exercises were not suitable or were being executed too-rapidly. It is this necessity that the patient should be under the observation of a properly trained clinical observer which makes it impossible that a treatment of this kind can ever safely be administered by a nurse-at any rate, until the patient is well advanced towards recovery, and it has been definitely ascertained what exercises he can stand with impunity. Then, and then only, it may be admissible to allow the movements to be performed without the presence of the medical attendant.

Among the advantages of the treatment of failure of compensation by mechanically resisted movements are the facts that (1) we do away with the expense of the nurse, which is often an important consideration in cases where only a certain sum can possibly be afforded to cover all medical treatment, \&c. ; (2) we keep the treatment in the hands of the medical practitioner, the time occupied being really so small that the treatment can easily be carried out during his daily visit; and (3) we substitute for the fallible muscles of a nurse the unvarying tension of a rubber cord.

Queen Anne-street, W.

The Whiteley Exerciser. 10, Ironmonger-lane, E.C

A MEETING of the Supreme Grand Chapter of Royal Arch Freemasons was held at Freemasons' Hall on Thursday, Aug. 5th, under the presidency of Mr. W. W. B. Beach, M.P. On the motion of Sir George D. Harris a donation of 100 guineas was voted to Guy's Hospital in aid of the special effort now being made on behalf of the institution.

\section{POISONING BY MAGNESIUM SULPHATE (EPSOM SALTS).}

BY J. HEADLEY NEALE, M.B. EDIN., M.R C.P.LOND., PHYSICIAN TO THE LEICESTER INFIRMARY AND FEVHR HOUSE.

ON the evening of April 26th I was asked by my friend $\mathrm{Mr}$. Montague Williams to see a lad aged fifteen years at the Midland station, to whom he had been called a few minutes previously, finding him in so critical a condition that he would not on his sole responsibility sanction his removal. to the infirmary. The history was as follows. Being constipated on the previous day he purchased a penny packet (one ounce) of Epsom salts at a neighbouring druggist's and took the whole of the contents. Only three slight motions resulted and towards evening he began to feel very sick and ill and remained so through the night. On the following morning his landlady would not allow him to keep his bed so he went to his work at the station as usual, but was too ill to do anything and went to lie down on one of the other boys' beds, vomiting frequently during the day, Mr. Williams not being sent for till nearly 6 P.M. 'The following describes the condition in which we found the lad. He was lying outside the bed in an attitude of flexion (occasionally varying his position by rolling on to his stomach), perfectly indifferent to his surroundings, but answering questions intelligently when smartly roused. He declared " he had taken nothing the day before but the packet of Epsom salts he got from __.'s." The face and hands were deeply cyanosed, the lips, eyelids, alæ nasi, and auricles being of a dark purple colour, and when the finger was drawn forcibly down the forearm the resulting white line was very slow in regaining its colour, while the conjunctivæ were intensely congested and the pupils dilated and unequal. The covered parts of the body presented a roseolous rash, and there was a zone of herpes zoster in the left sub-mammary regior extending round to the back. During the few minutes we stood by his bedside the boy had several attacks of tetanic spasms, affecting the right side of the face and passing down the right arm, together with pronation of the hand. The tongue and teeth were covered with "sordes," the stomach was enormously dilated, and his urine was dribbling away. The right radial pulse was absent and the left hardly perceptible; the heart beats were feeble and could not be counted. The extremities were cold the axillary temperature was $105^{\circ} \mathrm{F}$. We gave the boy a stiff dose of hot brandy-and-water, bad him placed on the station axabulance well covered with blankets and packed with hot-water bottles, and he was at once removed to the infirmary, the immediate treatment on arrival being a draught containing carbonate of ammonia spirits of ether, and tincture of strophanthus. On paying my usual visit the following morning $\bar{I}$ found that the patient had had frequent vomitings of a greenish fluid. The cyanosis was less marked, the radial pulses were perceptible, and he was altogether better and more conscious of his surroundings. Further examination, however, disclosed that his bladder was greatly distended. $\mathrm{He}$ was immediately catheterised and about forty ounces of urine drawn off. By the end of the week he was practically well and was discharged in a few days completely recovered.

I purchased at the earliest moment a packet of the salts which I was assured by the druggist came from the same cask, and through the kindness of Dr. Murrell, who took a great interest in the case, had it analysed by Dr. Wilson Hake, Professor of Chemistry at the Westminster Hospital Medical School, whose report is a follows:-_" I have care fully examined the sample of 'purified Epsom salts' from Mr. —_, chemist, Leicester, handed to me by Dr. Murrell in a sealed packet. I find it to be free from all poisonous metals, oxalic acid, or strychnine in any approximate quantity, but I have not looked for minute traces; it contains a small quantity of chloride of sodium and some potassium. It is, therefore, what it is labeled-namely, 'Purified Epsom Salts,' and abundant evidence of magnesium and sulphuric acid is to hand. It is also quite neutral in reaction and very soluble in water."

Remarks.-I was not told a single word of the history of the case until I had examined the boy, and I came to the conclusion that he was suffering from some irritant poison. This exactly tallied with Mr. Williams's previously formed 
opinion, and I was then acquainted with the story of the packet of Epsom salts. The only thing that suggested itself was "oxalic acid" by mistake, but we at once saw that had a similar quantity of that salt been taken twenty-four hours previously there would have been no necessity for a consultation over the case. Dr. Murrell reminded me that Dr. Luff had recorded a case where death had resulted in an adult male from taking a similar quantity. An interesting feature in the case was that the salts failing to act as a purge produced toxic effects; another the stimulation of certain motor centres, as evidenced by the tetanic spasm of the face and arm occurring coincidently with paralysis of the inbibitory fobres of the pneumogastric to the heart, paralysis of the muscular fibres of the stomach and bladder; and of the muscular coats of the bloodvessels, demonstrated by the absence of the radial pulse and general cyanosis.

Leicester.

\section{d.}

\section{HOSPITAL PRACTICE, BRITISH AND FOREIGN.}

Nulla autem est alia pro certo noscendi via, nisi quamplurimas et morSorum et dissectionum historias, tum aliorum tum proprias collectas babere, et inter se comparare--MoRGAGN De Sed. et Caus. Morb., lib. iv. Proœmium.

\section{NORWOOD COTTAGE HOSPITAL.}

CASE OF SECOND OVARIOTOMY AT THE AGE OF SEVENTY YEARS ; RECOYERY.

(Under the care of Dr. JoHN H. GALTON.)

$I_{N}$ the case of ovariotomy reported below there are two points worthy of notice-one is the fact that it was the second ovariotomy performed on the same patient and the other is the age of the patient. Formerly it was more commonly necessary to open the abdomen a second time to remove the other ovary some years after the first ovariotomy; now it is more customary to remove at an ovariotomy the second ovary also if there is any sign of its being diseased. There seems to be a tendency to the subsequent degeneration of the sound ovary after the diseased one has been removed. It is advisable to remove the second ovary at the same time as the first in all women who have passed the climacteric or who are closely approaching it, provided, of course, that this addition to the operation occasions no additional risk to the patient. The presence of a large uterine fibroid would also be an indication for removal of the second ovary. With regard to the second point-namely, the age of the patient-it may be noticed that age seems to be no contraindication to the operation. Many instances have been recorded of cases of ovariotomy above the age of seventy years. J. Rutherford Morison ${ }^{1}$ of Newcastle-on-Tyne recorded five cases all over seventy years of age, and of these only one died. Homans ${ }^{2}$ (of Boston, U.S.A.) recorded twelve cases, of whom three died. Mary Sherwood ${ }^{3}$ (of Baltimore) collected thirty-eight cases with five deaths. Spencer Wells's oldest case was seventy-one years of age. Keith's oldest case was seventy-three years of age. Terrien removed both ovaries from a woman aged seventy-seven years. ${ }^{4}$ Several cases have been recorded of ovariotomies on patients over eighty years of age. Schroeder's case ${ }^{5}$ was eighty years of age, and this was also the age of a case operated on by Owens. ${ }^{6} \mathrm{Edis}^{7}$ records a case over the age of eightyone years. H. Spencer's case comes next, ${ }^{8}$ and the age here was eighty-two years and three months. Homan's case? was one month older; but J. Paul Bush (of Bristol) has recorded a case ${ }^{10}$ in which ovariotomy was performed on a patient who had commenced her eighty-fifth year. It is very sotisfactory to be able to add that all these patients over eighty years of age recovered from the operation.

A woman aged seventy years, upon whom Dr.

I ThE LANCET, Jan. 21st, 1893.

2 Boston Medical and Surgical Journal, May 25th, 1893. 3 Clinical Record, Philadelphia, July, 1893. 4 Le Progrès Médical, July 16th, 1891.
5 Olshausen, Krankheiten der Orarien. 6 Journal of the British Gynecological Society, rol, iv. 7 Ibid., vol. viii. o Brit. Med. Jour., Dec. 9th, 1893. 9 Loc. cit. 10 Brit. Med. Jour., July 14th, 1894
Galton performed an ovariotomy twelve years ago, a report of which appeared in THK LANCET of Dec. 27th, 1884, was admitted to Norwood Cottage Hospital on Jane 16th, 1896. She had been quite well till six months before, when she first noticed enlargement of the abdomen, which lad gradually increased. On her admission the abdomen measured $35_{2}^{\frac{1}{2}}$ inches round the waist and 39 inches round the umbilicus. While under observation for ten days these measurements were found to vary from $32 \frac{1}{2}$ and $39 \frac{1}{2}$ inches to $35 \frac{1}{2}$ and $35 \frac{3}{4}$ inches. The features were shrunken and the countenance was anxious. The abdomen was duil to a level of two inches above the umbilicus, the line of dulness varying slightly and resonant in both flanks. Iluctuation was perceptible throughout, but there was a solid feel in the tumour in the right iliac region. Per vaginam a bulging on the left side of the uterus was felt. On July 2nd at 12 o'clock noon, Dr. Shearer administered chloroform and Mr. Sidney Turner and Mr. Arthur Turner assisted. An incision of two and a half inches was made an inch to the left of the scar of the former operation through the left rectus muscle. On opening the peritoneum some clear fluid escaped and the tumour was found strongly adherent to the anterior abdominal wall and still more strongly to the brim of the pelvis and the anterior surface of the uterus. Dark coffee-ground fluid escaped on puncturing the larger cyst and thick white opaque fluid from the smaller and deeper cysts. To make space for dealing with the adhesions and facilitate the removal of the more solid portions of the tumour the abdominal incision was enlarged one inch. The pedicle was then isolated, transfixed, and tied in double ligature crossed. The adhesions were then separated, only one ligature being required for the deep adhesion, the tumour was removed, and the abdomen washed out with boiled water at $110^{\circ} \mathrm{F}$., and the wound closed by five deep silrer sutures and five superficial ones and dressed with boracic acid in powder and pads of alembroth gauze. The tumour weighed $1 \mathrm{lb} .14 \mathrm{oz}$. solid and $240 \mathrm{oz}$. fluid. 'The operation occupied one hour. On removal to bed the pulse-rate was 68 and the temperature $974^{\circ}$. She was ordered one drachm of hot water every hour. At 9 P.M. the pulse was 76 and the temperature $98.8^{\circ}$, and nine and three-quarter ounces of urine were drawn off at midnight The voice was strong. On the 3 rd the pulse was 86 and the temperature $98.8^{\circ}$. The patient passed nine and a quarter ounces of urine naturally at 9 A.M. At 10 P.M. the pulse was 90 and the temperature $984^{\circ}$, and she complained of thirst. She was ordered ice to suck and half an ounce each of milk and barley-water every hour, and one drachm of whisky in milk twice during the night. On the 4th the pulse-rate was 82 and the temperature was $98 \cdot 6^{\circ}$, and she was ordered one ounce of beef-tea every alternate hour during the day and one drachm of whisky in milk every four hours. On the 5 th the pulse was 82 and the temperature $98^{\circ}$. She was given pish pash (rice boiled with meat) twice daily and tea and bread and butter. The whisky and milk were given every three hours. The patient passed flatus. On the 6 th the pulse was 80 and the temperature $98 \cdot 1^{\circ}$. The wound was dressed and looked quite healthy, the dressing being only stained from slight blood oozing. At 7 P.M. the temperature was $100 \cdot 1^{\circ}$, and at 10 P.M. $100 \cdot 8^{\circ}$. On the 7 th the pulse was 80 and the temperature was $99.1^{\circ}$, and the patient was given nish for dinner and half an ounce of compound liquorice powder at night. On the 8 th the pulse was 84 and the temperature $98 \cdot 2^{\circ}$. The bowels not having acted, she was given an enema consisting of one drachm of glycerine and six drachms of hot water. The bowels acted at 6 P.M. On the 9 th the pulse was 80 and the temperature was $98^{\circ}$. The wound was dressed and all the deep and superficial sutures removed. She was ordered chicken. The wound had all healed with the exception of half an inch in the centre, where there was an overlapping edge of skin to which a small pad was applied. On the 10th the pulse was 78 and the temperature $97 \cdot 8^{\circ}$. On the 11th the pulse was 72 and the temperature $98^{\circ}$. The whisky was omitted and four ounces of port wine daily were ordered. On the 12 th the pulse was 76 and the temperature $97.4^{\circ}$, and on the 13th the wound had quite healed. On the 16: h an abdominal belt was applied. The patient went on quite well and was discharged from hospital on the 27th.

Remarts by Dr. GALTON.-At the first operation twelve years ago the patient was so collapsed that the necessity for rapidly closing the abdomen did not admit of examination or remoral of the remaining ovary. The second operation, though fully as difficult as the first owing to adhesions, was better borne in spite of the patient being seventy years of 